\title{
Enhanced anti-glioblastoma activity of microglia by AAV2-mediated IL-12 through TRAIL and phagocytosis in vitro
}

\author{
TSUNG-LANG CHIU ${ }^{1,2}$, CHIH-WEN PENG ${ }^{3}$ and MEI-JAN WANG ${ }^{4,5}$ \\ ${ }^{1}$ Tzu-Chi University; ${ }^{2}$ Division of Neuro-Oncology, Neuro-Medical Scientific Center, Buddhist \\ Tzu-Chi General Hospital; ${ }^{3}$ Department of Life Science, Tzu-Chi University; ${ }^{4}$ Department of Research, \\ Buddhist Tzu Chi General Hospital; ${ }^{5}$ Tzu Chi College of Technology, Hualien, Taiwan, R.O.C.
}

Received December 15, 2010; Accepted February 8, 2011

DOI: 10.3892/or.2011.1213

\begin{abstract}
Microglia have been found to infiltrate into malignant brain tumors. However, their function is usually reversed by cancerous cells thus contributing to cancer growth and metastasis. We propose that microglial immuno-activity can be modulated with interleukin-12, by which the anti-cancer ability might be restored. To this end, a strategy was designed using AAV2 carrying interleukin-12 to activate microglia to eliminate cancerous cells. Under this strategy, recombinant AAV2 encoding interleukin-12 was constructed and evaluated for its transduction efficacy on cancerous and CNS cells. The bioactivity of microglia modulated by interleukin-12 was examined and death receptors 4 and 5 were detected on cancerous cells. The effects of interleukin-12 on microglial cytotoxicity were evaluated by MTT assay. The human cell line DBTRG, surgical specimens of GBM and rat astrocytes expressed AAV2-mediated GFP quite strongly. Interleukin-12 secretion was detected in DBTRG, RG2 and astrocytes after the transduction of AAV2 encoding interleukin-12. TRAIL releasing and phagocytotic activity of microglia were significantly increased after interleukin-12 stimulation. MTT assay of microglial cytotoxicity elicited significant increase after the stimulation with interleukin-12 protein. In conclusion, AAV2 is an effective vector in transferring therapeutic genes such as interleukin-12 to enhance microglial anti-cancer activity and to eliminate cancerous cells.
\end{abstract}

\section{Introduction}

Among the reports of recent anti-cancer research, immunomodulation with cytokines is one of the modalities exhibiting

Correspondence to: Dr Tsung-Lang Chiu, Division of NeuroOncology, Neuro-Medical Scientific Center, Buddhist Tzu-Chi General Hospital, 707, Section 3, Chung-Yang Road, Hualien, Taiwan, R.O.C.

E-mail:poluschiou@gmail.com

Key words: adeno-associated virus, glioblastoma multiforme, microglia, interleukin-12, TRAIL the most therapeutic potential (1-4). Many pro-inflammatory cytokines have been investigated and clarified for their anticancer effects and mechanisms. Among those, interleukin-12 (IL-12) discloses the most power in enhancing either innate or adaptive immune-responses for anti-cancer activity. In the cascade of IL-12 activity, the antigen presenting cells, such as dendritic cells, macrophage and neutrophil, produce and secrete IL-12 after encountering the invading pathogen or transformed cells. Subsequently, IL-12 can induce proliferation of hematopoietic progenitor cells, generation of IL-3 and stem-cell factors, maturation of natural killer $\mathrm{T}$ cell and cytotoxic $\mathrm{T}$ cell, and increasing of interferon- $\gamma$, granulocytemacrophage colony-stimulating factor, tumor necrosis factor and IL- 8 . In addition, IL-12 may facilitate the $\mathrm{T}_{\mathrm{H}} 1$ pathway to promote naïve $\mathrm{T}$ cells developing to memory $\mathrm{T}_{\mathrm{H}} 1$ cells (5). In anti-cancer activity, IL-12 has been demonstrated by stimulating or promoting the functions of NK cells or T cells to enhance anti-angiogenesis through secreting of interferon- $\gamma$ and to induce apoptosis of tumor cells through secreting of the members of tumor necrosis factor (TNF) super-family. Furthermore, IL-12 may enhance tumor antigen-specific adaptive immunity through the $T_{H} 1$ pathway (6). Nevertheless, the adverse effect of systemic administration of pro-inflammatory cytokines emerged from the human clinical trail of IL-12 protein is extremely harmful (7). In addition, from the experience of clinical practice, malignant brain tumors always recur or relapse within a short period after the initial treatment, even in the cases of invisible tumor in post-therapeutic imaging study (8-10). Therefore, a superior method should be designed as a long-term expression of cytokines and local tissue affection for the purpose of maximal therapeutic effectiveness and restricted systemic toxicity.

For the purpose of long-term expression and local delivery of cytokine, an effective vehicle to transfer the cytokine into the focal tissue is superior to direct protein injection or naked DNA injection. In the choice of a mediating vector, accumulating evidence has verified the feasibility of adenoassociated virus (AAV) in genomic transportation $(11,12)$. AAV is of the parvovirus family and is characterized by being deficient in replication, non-pathogenic to humans, and able to transfect most cell types (13). The genes mediated by AAV keep expressing up to 15 months and sustain in a high level during the 2 nd to 4 th month when inoculated in the mouse 
brain (14). Its competence and safety have been elucidated in several clinical human trials since early 1996, when cystic fibrosis was converted with AAV2 transferring the cystic fibrosis transmembrane conductance gene into lung tissue. In 2007, advanced Parkinson's disease was treated with AAV2 transferring the glutamic acid decarboxylase gene into the subthalamic nucleus $(15,16)$.

Microglia, the central nervous system (CNS) immune cells, comprise $2-20 \%$ of the population of CNS cells, which execute the defense function against invading microbes and the clearance of transformed cells $(17,18)$. In a tumor environment, microglial cells infiltrate the tumor more than other peripheral immune cells and have been estimated to be up to $10-34 \%$ of the composition of tumor mass (19). Microglial cells represent the antigen presenting function in CNS through major histocompatibility complex type 2 and the phagocytotic ability. Furthermore, they generate cytokines, including IL- $1 \alpha$, IL- $\beta$, IL-6, IL-12, IL-16, IL-23, TNF- $\alpha$ and transforming growth factor and chemokines, including MCP-1, MIP-1, IL-8, MIG and IP-10 (20,21). However, the immune performance of microglia in the tumor environment was diversely and continuously affected by the tumor-released factors, such as immunosuppressive cytokines, resulted in the loss or reduction of their antigen presenting activity and innate immune response $(22,23)$. Furthermore, the extracellular matrix protease released by microglia, which infiltrated into the tumor and was changed in behavior by the tumor-released factor, was postulated to promote the invasion and migration of tumor cells (24). Therefore, readjusting or reinforcing the native immune activity of tumor-infiltrating microglial cells will achieve better anti-cancer activity than the peripheral immune cells by disrupting the tumor-promoted effects and restoring the anti-tumor ability of the abundant tumor-infiltrating microglial cells. In this study, we constructed AAV type 2 (AAV2) encoding IL-12 to survey the expressive efficiency of astrocyte, microglial cell and glioblastoma multiforme (GBM) cell and to judge the feasibility of AAV2 in malignant brain tumor therapy. Further, owing to the high composition of microglial cells in tumor mass, we evaluated the anti-tumor effect of microglia pre- and post-stimulation with IL-12 on account on its secretion of TNF related apoptosis inducing ligand (TRAIL) and also its phagocytotic activity.

\section{Materials and methods}

Construction of AAV2 encoding IL-12. The plasmid pORFmIL-12 (p40:p35) (InvivoGen, San Diego, CA, USA), 5'-primer: mIL-12-F (GAATTCATGGCCTGTCCTCAGA AGCT) and 3'-primer: mIL-12-R (GGATCCTTAGGCG GAGCTCAGATAGC), was amplified using polymerase chain reaction (PCR) and subcloned into pAAV-MCS (Stratagene, La Jolla, CA, USA), then verified by DNA sequencing (Protec, Oberstenfeld, Germany). PCR was carried out in a PerkinElmer Cetus thermocycler. The primers were denatured at $94^{\circ} \mathrm{C}$ for $45 \mathrm{sec}$, annealed at $60^{\circ} \mathrm{C}$ for $45 \mathrm{sec}$, and extended 30 cycles at $72^{\circ} \mathrm{C}$ for $2 \mathrm{~min}$ then $72^{\circ} \mathrm{C}$ for $10 \mathrm{~min}$. The production of AAV2 encoding IL-12 (AAV2/IL-12) included 5 main steps; transformation of $E$. coli, plasmid extraction, culture of HEK 293 cells, package of AAV2 in 293 cells and AAV2 purification (25). In brief, E. coli (ECOS 101) cells were used as competent cells and transformed by pAAV-RC (Stratagene), pHelper (Stratagene), pAAV-IL-12, which were cultured with 2YT-Broth A50 (Invitrogen, Carlsbad, USA) medium containing ampicillin $(50 \mu \mathrm{g} / \mathrm{ml})$ at $37^{\circ} \mathrm{C}$. The transformed $E$. coli were resuspended in 2YT-Broth medium A50 and prepared for extraction of the three plasmids. The plasmid DNAs were purified with a plasmid Mega preparation kit (Qiagen, Duesseldorf, Germany). Each of the extracted plasmids was quantified with OD260/280. The frozen HEK 293 cells were thawed under $37^{\circ} \mathrm{C}$ and cultured with DMEM (Cellgro, Manassas, USA), 10\% FBS (Hyclone), and 1\% penicillin/streptomycin (Gibco, Carlsbad, USA). The extracted plasmids, pAAV-RC, pAAV-IL-12 and double the pHelper, were mixed and shaken with $\mathrm{CaCl}_{2}$. The mixture was spread into HEK 293 cell culture dish and incubated with DMEM, $10 \% \mathrm{FBS}$, and $1 \% \mathrm{P} / \mathrm{S}$ at $37^{\circ} \mathrm{C}$ for AAV-GFP package. The packaged AAVs were purified through heparin column (Heparin Actigel Sterogen, San Gabriel, USA).

Transfection of rAAV2 on targeted cells. Five cell types, human GBM cell line DBTRG, rat GBM cell line RG2, murine microglial cell line BV2, primary culture of rat astrocyte, and primary culture of human GMB cells, were surveyed for rAAV2 transductive efficacy. The RG2, DBTRG and BV2 cell line were purchased from the Food Industry Research and Development Institute (Hsinchu, Taiwan). The astrocytes were extracted from 1-day-old Spraque-Dowley rats (26). Human GBM cells were cultured from a surgical specimen proven by a pathologist. Briefly, the specimen was washed with PBS solution and cut into pieces. After centrifugation at 12000 rpm for $5 \mathrm{~min}$, the pellets were mixed with $2 \mathrm{ml}$ trypsin/ EDTA solution and shook in $37^{\circ} \mathrm{C}$ water bath for $10 \mathrm{~min}$. The suspension was added in $3 \mathrm{ml}$ DMEM and filtered through a $70-\mu \mathrm{m}$ filter into a 50-ml tube. After centrifugation, the pellets were mixed with $12 \mathrm{ml}$ DMEM then transferred to a $10-\mathrm{cm}$ culture dish and incubated at $37^{\circ} \mathrm{C}$ in a humidified incubator with $5 \% \mathrm{CO}_{2}$.

The targeted cells were spread into each well of 6-well plate and cultured with 10\% FBS (Hyclone), DMEM (Gibco, Manassas, USA), and penicillin-streptomycin sulfate (Gibco) for $24 \mathrm{~h}$. AAV2-GFP $(100 \mu \mathrm{l})\left(4.28 \times 10^{10} \mathrm{viral}\right.$ particles $\left./ \mathrm{ml}\right)$ was added in and inspected daily under fluorescent microscope to the fourth day. The expression of GFP was evaluated and pictured at $24 \mathrm{~h}$ and at four days after the addition of rAAV2. The same procedure was practiced on the other cell types.

ELISA assay of IL-12 expression. Five cell types, rat GBM cell line RG2, primary culture rat astrocyte, rat microglial cell line BV2, human GBM cell line DBTRG and HEK 293 (positive control), were transduced by AAV2/IL-12 and were evaluated for IL-12 expression. Cells $\left(1 \times 10^{5}\right)$ of each cell type were transferred to 6 wells of a 96-well plate and cultured with $10 \%$ FBS, DMEM and penicillin-streptomycin sulfate for $24 \mathrm{~h}$. AAV2/IL-12 in three conditions, $1 \times 10^{10}, 1 \times 10^{11}$ and $1 \times 10^{12}$ viral particles $/ \mathrm{ml}$, were added into cultured cells with one condition for two wells and were incubated for 4 days. The supernatant of each well was aspirated and span, then the upper clear fluid was obtained for estimating the mIL-12 concentration by ELISA Kit (M1270 Quantikine, 
Minneapolis, USA). The same procedures were performed with AAV2/GFP instead of AAV2/IL-12 for negative control.

ELISA assay of microglia-secreted cytokines. BV2 cells $\left(5 \times 10^{4}\right)$ were transferred to each well of a 96-well microplate then cultured with $2 \%$ FBS for $24 \mathrm{~h}$. The experiment was divided into three groups, non-stimulated, IL-12 (eBioscience, San Diego, USA)-stimulated and LPS (Sigma, St. Louis, MO, USA)-stimulated. Therefore, $100 \mathrm{ng}$ IL-12 and LPS were added into 18 wells individually to active the BV2 cells. After 24,48 and $72 \mathrm{~h}$ of stimulation, the supernatant was aspirated and collected for detection of TRAIL. The amount of TRAIL was detected with rat anti-mouse TRAIL/TNFSF10 monoclonal antibody as the primary antibody and biotinylated anti-rat IgG antibody as the secondary antibody (Quantikine $R \& D)$.

Western blot assay of TRAIL receptor. A total of 6 human cancer cell types, GBM cell lines DBTRG, G5T-VGH, GBM 8401, GBM 8901, along with pathologically verified GBM specimen nos. 3 and 4 , were evaluated by SDS-PAGE for death receptors 4 (DR4) and 5 (DR5). The protein was extracted with lysis buffer PRO-PREP (iNtRon, Rehovot, Israel). Before that, the tissue was ground with ceramic zirconium oxide beads. The protein concentration was determined by BCA assay read by ELISA reader (Molecular Devices, Silicon Valley, USA) at OD $590 \mathrm{~nm}$. The protein was analyzed on $10 \%$ polyacrylamide gel by SDS-polyacrylamide gel electrophoresis and transferred electrophoretically to nitrocellulose membranes at $400 \mathrm{~mA}$ for $2.5 \mathrm{~h}$. Goat anti-human TRAIL R1 and $R 2$ antibodies (R\&D) were used as primary antibody. Rat anti-goat IgG couple with horseradish peroxidase was used as secondary antibody and the activity of peroxide was detected with enhanced chemiluminescence.

Phagocytosis of microglia. BV2 cells were cultured in a $10-\mathrm{cm}$ culture dish with 10\% FBS + DMEM. The phagocytotic analysis was done using phagocytosis assay kit (Molecular Probes, Inc., Eugene, USA). Briefly, 4x105 BV2 cells were transferred into a total of 18 wells and incubated with $10 \%$ FBS + DMEM. Six wells without BV2 cells were added in $150 \mu \mathrm{l}$ of DMEM as negative control. Eighteen wells were equally divided into IL-12-treated, LPS-treated and non-treated groups. The stimulating reagent $100 \mathrm{ng}$ IL-12 or $100 \mathrm{ng}$ LPS was added and the culture medium was changed to $2 \%$ FBS + DMEM. After $18 \mathrm{~h}$ of incubation, $100 \mu \mathrm{l}$ of prepared $E$. coli fluorescent BioParticle suspension was added into each well then incubated for $2 \mathrm{~h}$. Trypan blue $(100 \mu \mathrm{l})$ was added and incubated for $1 \mathrm{~min}$ after removing the BioParticle loading suspension by vacuum aspiration. The fluorescence was read at $\sim 480 \mathrm{~nm}$ excitation and $\sim 520 \mathrm{~nm}$ emission. The phagocytotic efficacy was estimated with the formula of $\%$ Effect $=$ Net Experimental Reading/Net Positive Reading $\mathrm{x}$ $100 \%$.

MTT assay of microglial cytotoxicity on RG2 cells. MTT [3-(4,5-dimethylthiazol-2-yl)-2,5-diphenyltetrazoliumbromide] solution $(5 \mathrm{mg} / \mathrm{ml})$ was diluted with PBS in a ratio of $1: 9$ and stored at $2-6^{\circ} \mathrm{C}$ for further utility. Two different culture modes were performed: the co-culture mode using a 96-well plate and the trans-well culture mode using a trans-well 96-well plate. Four conditions were set in the co-culture mode, which included $2 \mathrm{ml}$ MTT medium only, $2 \mathrm{ml}$ MTT medium $+5 \times 10^{4}$ RG2 cells, $2 \mathrm{ml}$ MTT medium $+5 \times 10^{4}$ RG2 cells $+5 \times 10^{4}$ $\mathrm{BV} 2$ cells, and $2 \mathrm{ml}$ MTT medium $+5 \times 10^{4} \mathrm{RG} 2+5 \times 10^{4}$ BV2 cells +100 ng IL-12. The same conditions were applied in the trans-well mode, however, RG2 cells were incubated on the bottom plate with $2 \mathrm{ml}$ MTT medium and BV2 cells were incubated on the upper plate with $2 \mathrm{ml}$ MTT medium. The plates were incubated in a $37^{\circ} \mathrm{C}$ incubator for $4 \mathrm{~h}$. After centrifugation of the plate at $2000 \mathrm{rpm}$ for $5 \mathrm{~min}$, the upper fluid of the wells was removed and $1 \mathrm{ml}$ DMSO was added. The plate was incubated for $5 \mathrm{~min}$ then transferred to the reader for measurement of absorbance at $590 \mathrm{~nm}$.

MTT assay of microglia on AAV2/IL-12-transfected RG2 cells. The procedures were the same as above except the experimental groups which were changed to $5 \times 10^{4} \mathrm{RG} 2$ cells, $5 \times 10^{4}$ AAV2/IL-12-transfected RG2 cells, $5 \times 10^{4} \mathrm{RG} 2+5 \times 10^{4}$ BV2 cells, 5x10 4 AAV2/IL-12-transfected RG2 cells $+5 \times 10^{4}$ BV2 cells in co-culture model and $5 \times 10^{4}$ RG2 cells, $5 \times 10^{4}$ AAV2/IL-12-transfected RG2 cells on the bottom plate and $5 \times 10^{4} \mathrm{BV} 2$ cells on the upper plate in the trans-well model.

Statistical analysis. The data are depicted as the mean value \pm standard deviation. Student's t-test was applied to examine the difference when only two groups were compared. For comparison of multiple groups, one-way ANOVA with Bonferroni post-test was applied. The difference was assumed to be significant at $\mathrm{p}<0.05$.

\section{Results}

GFP expression of targeted cells. Human GBM cell line DBTRG, rat GBM cell line RG2, murine microglial cell line $\mathrm{BV} 2$, primary culture of rat astrocyte and primary culture of human GMB cells were transduced by AAV2/GFP. The five targeted cell types could be transduced by AAV2/GFP and expressed GFP after 4 days of incubation. In cancer cells, the expression of GFP of human GBM cell line DBTRG and GBM cells from excised specimen were near $100 \%$, however, the RG2 cells displayed scatter expression of GFP (Fig. 1). In CNS cells, the rat astrocytes illustrated good expression of GFP as well as the DBTRG, but the BV2 cells showed a few cells expressing GFP (Fig. 1).

Detection of secreted IL-12 from the targeted cells. RG2 cells, rat astrocytes, BV2 cells, DBTRG cells and HEK 293 cells were tranduced with AAV2/IL-12 and the secretion of IL-12 from five cell types were measured by ELISA. The DBTRG cells exhibited the highest ability, even greater than HEK 293 (positive control) in the secretion of IL-12 after transduction of rAAV2/IL-12. The astrocytes and RG2 cells also expressed IL-12 in abundant amounts. Nevertheless, BV2 cells did not express detectable IL-12 after four days of transduction. The five cell types that were transduced with AAV/GFP as negative control displayed no detectable IL-12 (Table I).

Cytokine secretion of microglia after IL-12 stimulation. Based on the above results, the human GBM cells and 


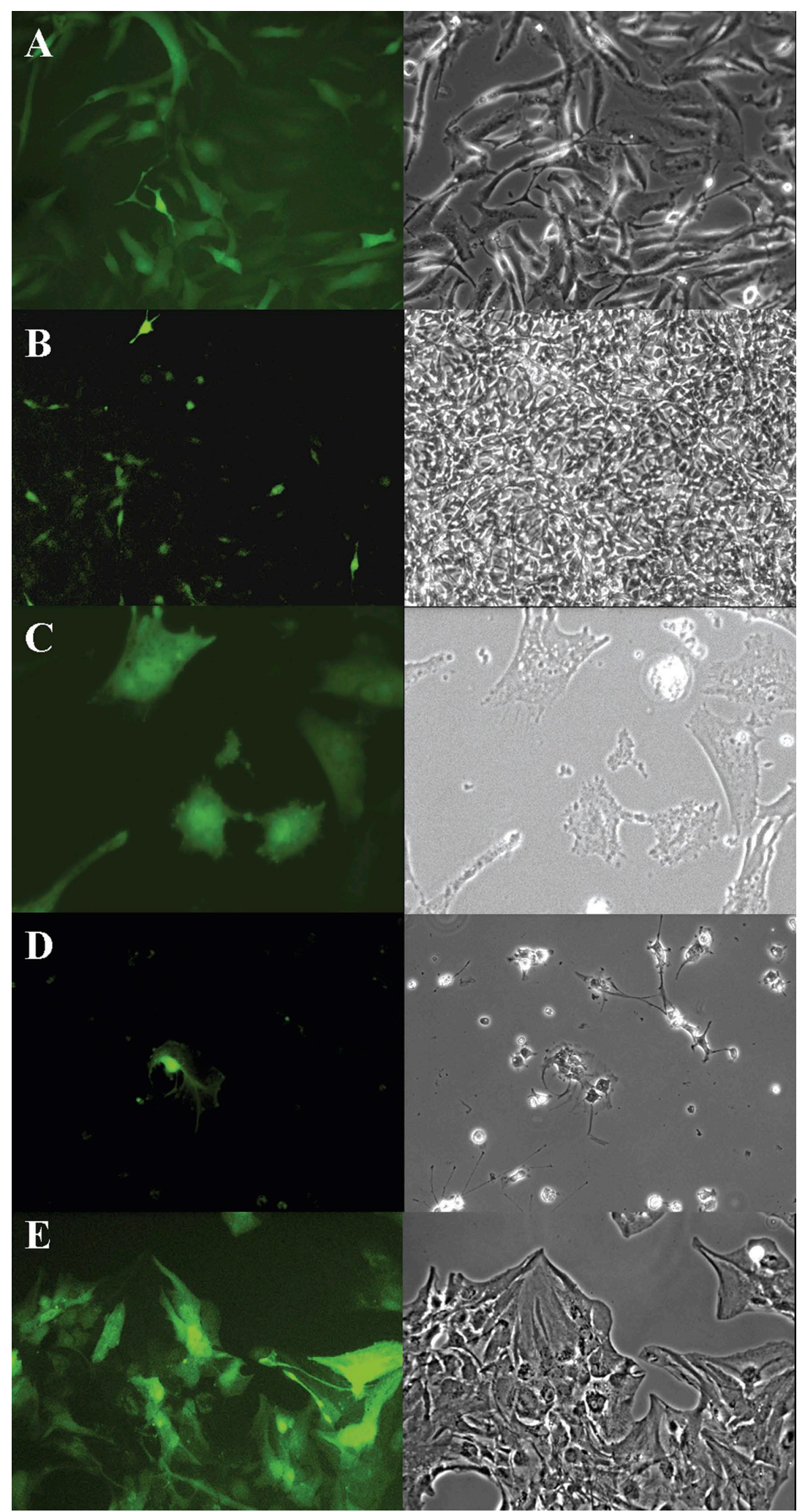

Figure 1. Cancerous cells and CNS cells after AAV2/GFP transduction. Photographs of GBM cell lines, surgical specimens of GBM, and CNS cells caught under microscope 4 days after being co-cultured with AAV2-GFP. The left photograph was pictured under a green fluorescent microscope and the right under phase contrast. (A) DBTRG cells were inspected under a green fluorescent microscope, left; under phase contrast, right (high power field, x400). (B) RG2 cells (low power field, x100) and (C) GBM cells from surgical specimen (high power field, x400). (D) BV2 cells (low power field x100). (E) Astrocytes (high power field $\mathrm{x} 400$ ).

astrocytes exhibit superior candidates for AAV2 transduction and secrete AAV2-mediated IL-12. However, the subsequent behavior of microglia after IL-12 stimulation is the critical pivot of the anti-cancer activity, so we examined the level of TRAIL which might secrete from microglia and immerge in the anti-cancer mechanisms. The amount of TRAIL was 
Table I. The amount of IL-12 secreted from CNS and GBM cells.

\begin{tabular}{|c|c|c|c|c|c|c|}
\hline & \multicolumn{3}{|c|}{ AAV-GFP IL-12 (pg/ml) } & \multicolumn{3}{|c|}{ AAV-IL-12 IL-12 (pg/ml) } \\
\hline & $10^{10} \mathrm{VP}$ & $10^{11} \mathrm{VP}$ & $10^{12} \mathrm{VP}$ & $10^{10} \mathrm{VP}$ & $10^{11} \mathrm{VP}$ & $10^{12} \mathrm{VP}$ \\
\hline BV2 & 0 & 0 & 0 & 0 & 0 & 0 \\
\hline Astrocyte & 0 & 0 & 0 & 365.50 & $>2000$ & $>2000$ \\
\hline HEK 293 & 0 & 0 & 0 & 1624.8 & $>2000$ & $>2000$ \\
\hline RG2 & 0 & 0 & 0 & 129.30 & 1000.8 & $>2000$ \\
\hline DBTRG & 0 & 0 & 0 & ${ }^{a}>2000$ & $>2000$ & $>2000$ \\
\hline
\end{tabular}

The IL-12 secreted from BV2, rat astrocyte, HEK 293, rat GBM cell line RG2 and human GBM cell line DBTRG were estimated 4 days after being co-cultured with AAV2/IL-12 in three different conditions: $1 \times 10^{10}, 1 \times 10^{11}$ and $1 \times 10^{12}$ viral particles/ml. The data are the mean of three wells in the same condition. ${ }^{\text {IIL-12 }} 12$ secretion from DBTRG was over the maximal value $(2000 \mathrm{pg} / \mathrm{ml})$ of estimating scale in all three AAV2 conditions.

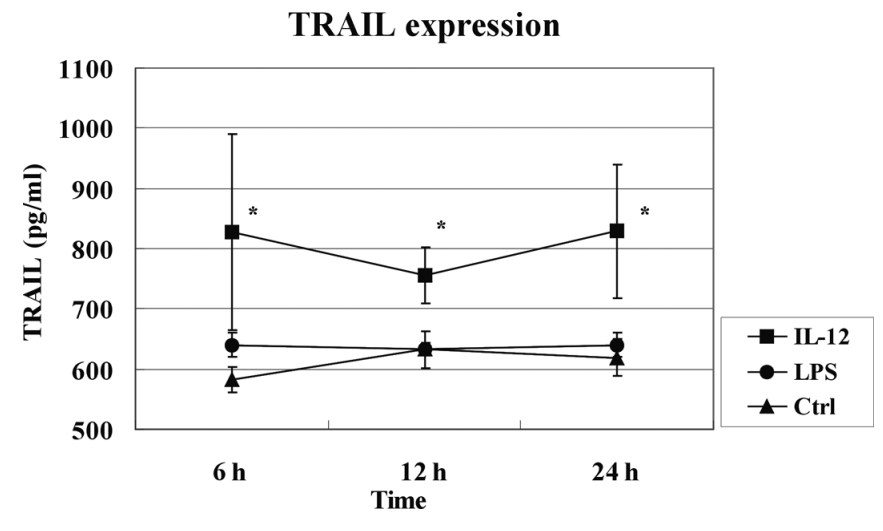

Figure 2. TNF- $\alpha$ secreted from BV2 cells. TNF- $\alpha$ was estimated with ELISA after $24 \mathrm{~h}$ stimulation of IL-12 protein. The control represented non-IL-12stimulating group.
$819.2 \pm 45.2 \mathrm{pg} / \mathrm{ml}$ after $6 \mathrm{~h}$ incubation, $805.1 \pm 45.6 \mathrm{pg} / \mathrm{ml}$ after $12 \mathrm{~h}$ incubation, and $869.9 \pm 67.8$ after $24 \mathrm{~h}$ incubation with IL-12. The LPS did not stimulate BV2 cells to secrete TRAIL as well as IL-12 since its level were $681.9 \pm 20.3 \mathrm{pg} /$ $\mathrm{ml}, 675.5 \pm 20.3 \mathrm{pg} / \mathrm{ml}$, and $686.3 \pm 10.7 \mathrm{pg} / \mathrm{ml}$ after 6,12 and $24 \mathrm{~h}$ incubation, respectively. The ANOVA analysis elicited significant differences between the three groups, and the IL-12-stimulated group exhibited significant differences with the LPS-stimulated and non-stimulated groups at the three time points (Fig. 2).

Expression of death receptor of tumor cells. Since TRAIL can be induced by IL-12 in microglia (BV2), we further interpreted the TRAIL receptors, DR4 and DR5, in GBM cells to elucidate the feasibility of anti-GBM by microglia through TRAIL pathway. All the 6 tested GBM cell types, DBTRG, G5T-VGH, GBM 8401, GBM 8901, GBM specimen no. 3 and

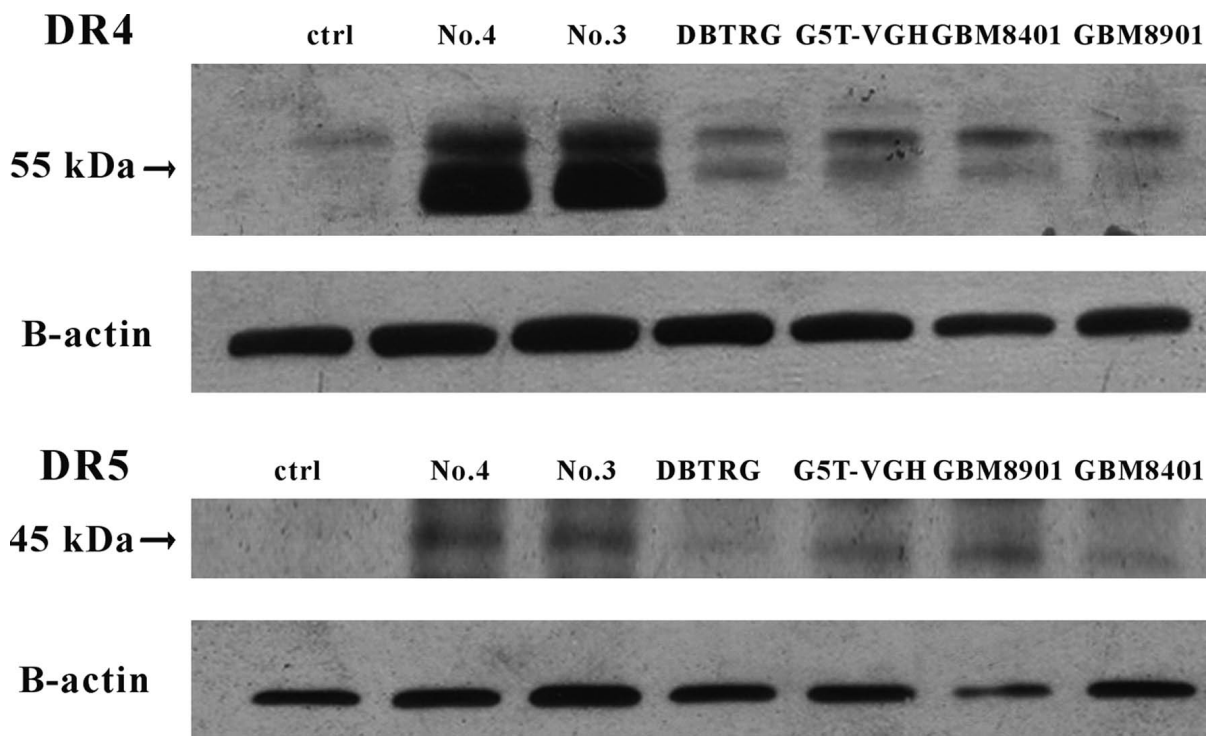

Figure 3. TRAIL secreted from BV2 cells. TRAIL was estimated with ELISA after the stimulation of IL-12 and LPS (positive control) at the time intervals of 6,12 and $24 \mathrm{~h}$. The control represented non-stimulating group. * $\mathrm{p}<0.05$, significant difference of IL-12-stimulated group compared with LPS and control at different time interval by ANOVA analysis. 


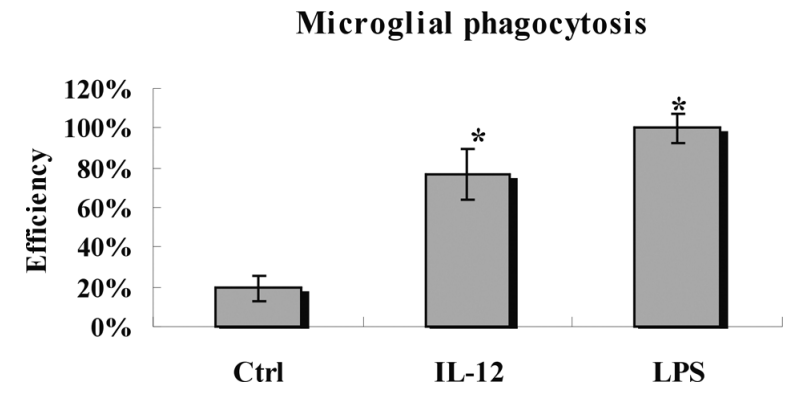

Figure 4. The Western blotting of DR4 and DR5 expressed on GBM cells. The proteins were extracted from human GBM cell lines DBTRG, G5T-VGH, GBM8401, GBM9801 and primary culture GBM cells 3 and 4 . The molecular weight is $55 \mathrm{kDa}$ for DR4 and $45 \mathrm{kDa}$ for DR5.

GBM specimen no. 4, expressed DR4 and DR5 by Western blot assay (Fig. 3).

Phagocytotic activity of microglia. In addition to TRAIL, the constitutionally phagocytotic activity of microglia was evaluated by using phagocytosis assay kit. The phagocytotic activity of BV2 was enhanced obviously by either IL-12 or
LPS. The phagocytotic efficacy was $76.7 \pm 12.9 \%$ in IL-12stimulated BV2 cells and 100.0 \pm 7.6\% in LPS-stimulated BV2 cells. The ANOVA analysis elicited significant differences between the three groups. The phagocytotic activity in BV2 cells of the IL-12-stimulated group exhibited significant differences with the non-stimulated groups (Fig. 4).

Cytotoxicity of microglia and IL-12-enhanced microglia on $R G 2$ cells. The cytotoxicity of BV2 on the rat GBM cells RG2 was evaluated by MTT assay. The IL-12-stimulated BV2 cells exhibited significantly increasing cytotoxicity on RG2 cells either in co-culture or trans-well model. In contrast, the non-IL-12-stimulated BV2 cells exhibited no significantly increasing cytotoxicity on RG2 cells in either model (Fig. 5). In the secondary MTT assay of microglial cytotoxicity, only the group of BV2 cells co-cultured with AAV2/IL-12-transfected RG2 cells displayed significantly increasing cytotoxicity. Differing from the experiment of IL-12 protein stimulation, the trans-well model of BV2 cells plus AAV2/IL-12-transfected RG2 showed no cytotoxic activity of the BV2 cells. This phenomenon was interpreted as showing that BV2 cells could not be stimulated by the RG2-secreted IL-12 since BV2 cells

MTT assay of RG2 survival

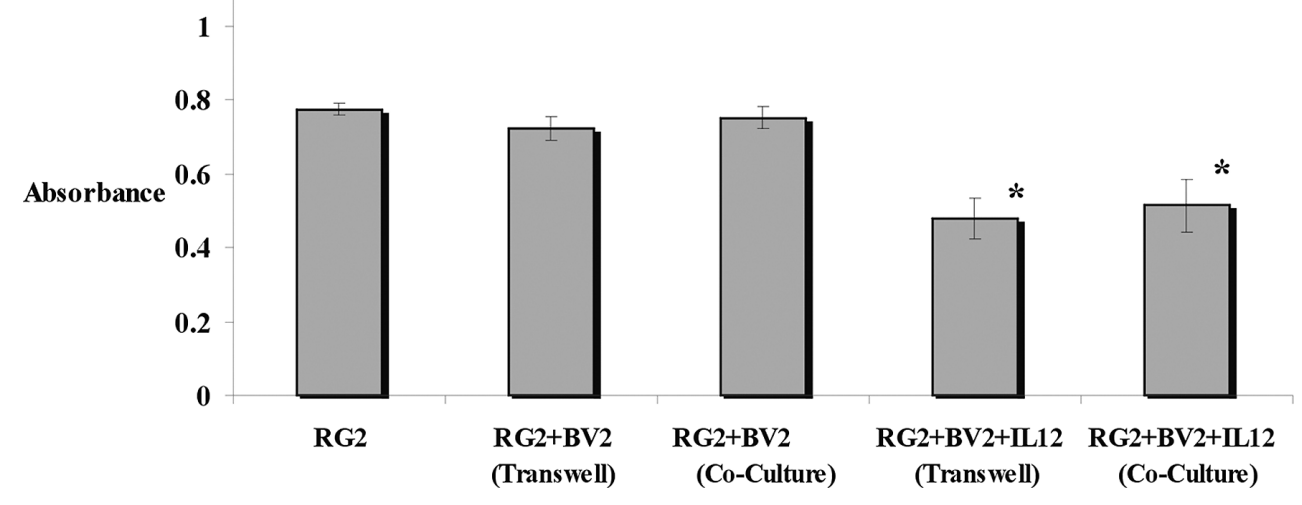

Figure 5. The phagocytotic activity of BV2 cells. The phagocytotic efficiency was estimated with fluorescent particles engulfed by BV2 cells in non-stimulated (control), IL-12 stimulation and LPS stimulation conditions. "P<0.05 versus control.

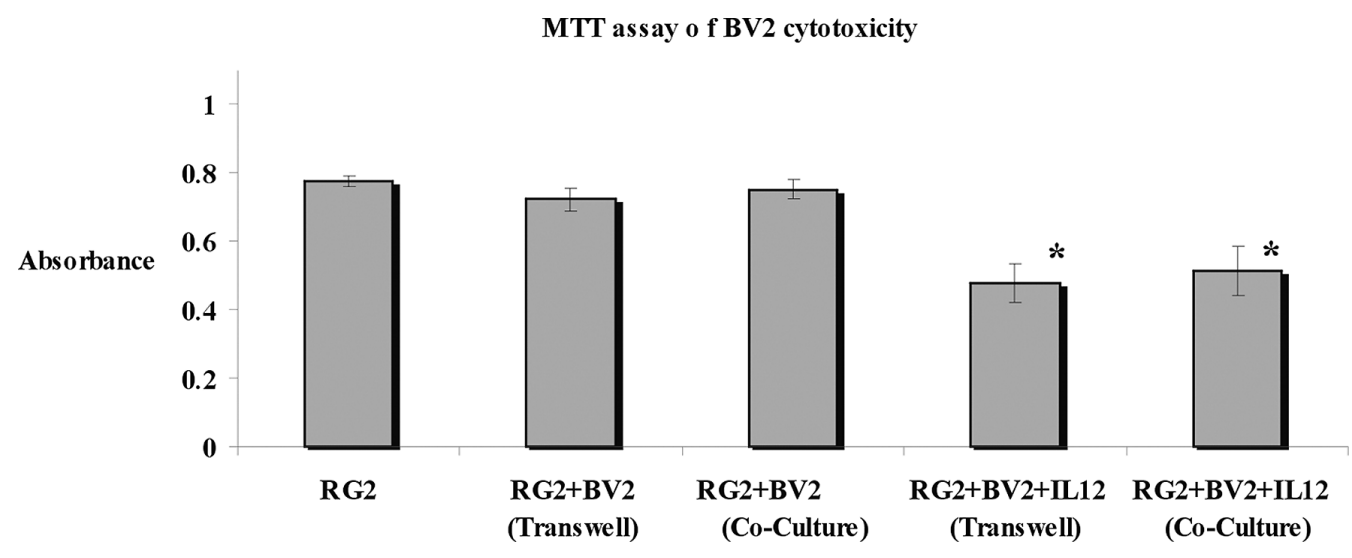

Figure 6. The cytotoxic effficacy of BV2 cells evoked by IL-12. The comparison of BV2 cytotoxicity on RG2 cells with and without stimulation of IL-12 protein was evaluated by MTT assay in trans-well and co-culture two different environments. The absorbance of the five groups, RG2, RG2 and BV2 co-cultured, RG2 in bottom well and BV2 in upper well, RG2 and BV2 and IL-12 co-cultured, and RG2 in the bottom well and BV2+IL-12 in upper well, were measured. ${ }^{*} \mathrm{p}<0.05, \mathrm{RG} 2+\mathrm{BV} 2+\mathrm{IL}-12$ in trans-well compared with RG2, RG2+BV2 in trans-well, or RG2+BV2 in co-culture; RG2+BV2+IL-12 in co-culture compared with RG2, RG2+BV2 in trans-well, and RG2+BV2 in co-culture. 
were incubated on the upper plate. Furthermore, the survival of RG2 cells exhibited no difference after being transfected by AAV2/IL-12 as compared with the non-transfected cells (Fig. 6).

\section{Discussion}

AAV has been verified as a competent vehicle to transfer therapeutic genes into many cell types of animals and humans $(11,27)$. However, the efficiency of transduction is variable and depending on the type of targeted cell and the serotype of AAV $(28,29)$. From the results of this experiment, the human GBM cells displayed remarkable AAV2-transductive efficacy in the expression of GFP and IL-12, while the rat GBM cells (RG2) disclosed weak transduction by AAV2. In CNS cells, the microglial cells (BV2) disclosed poor transductive efficiency by AAV2 carrying either GFP or IL-12. Therefore, it is important to elucidate the transductive efficiency of AAV-targeted cells, cancer cells or tumor-infiltrated cells, if AAV is to be considered as a vector carrying therapeutic genes. On account of this study, the hypothesis for treatment of malignant brain tumor by local AAV2 delivery presumes to follow the sequential events: cancer cells tranducted by AAV2/IL-12, cancer cells expressed IL-12 to induce or enhance the cytotoxicity of tumor-infiltrating microglial cells, and activated microglial cells eliminated cancer cells. In this strategy, the undesired side-effects from excessive IL-12 will be reduced when IL-12-secreting cancer cells are destroyed by microglial cells, since the microglial cells do not influence AAV2-IL-12 to secrete IL-12.

Cytokines secreted from malignant brain tumor cells, such as interleukin-6, leukemia inhibitory factor, oncostatin-M, and tumor growth factor- $\beta$, contribute to survival, proliferation and migration of tumor cells and inhibition of immune responses (24). By means of genetic transduction of AAV2, the secreted factors of tumor cells could be transferred to dominance in cancer-defense. In this study, the human GBM cells expressed abundant IL-12 after transduction of AAV2 encoding IL-12, which would induce the immune cascade of IL-12 in tumor cells or microglia.

Microglia, the resident immune cells in CNS, constitutively represent antigen-presenting and innate immunity have been found infiltrating malignant brain tumors. In accumulating evidence, however, their functions are converted from cancer-defense to cancer-contribution by cancerous cells or cancerous microenvironment $(24,30,31)$. Although, in this experiment, microglia could not be transfected by AAV2-IL-12 to express IL-12, its constitutive bioactivity could be induced or enhanced by administration of IL-12. Two provoked anti-cancer bioactivities of microglia, TRAIL and phagocytosis, were demonstrated in this study. TRAIL is a cell death-inducing ligand, which has found to secrete mainly from activated microglia in CNS and related to the degenerative and inflammatory diseases (32-34). TRAILinduced apoptosis is highly dependent on the TRAIL receptor presented on cancer cells (35), so we explored the DR4 and DR5 in four human GBM cell lines and two excised GBM specimens. All of the examined GBM cells express DR4 and DR5, confirming the feasibility of anti-cancer therapy by IL-12 stimulating microglia through the TRAIL pathway. Phagocytosis is one of the major functions of microglia to eradicate the invading pathogen and damaged cells, but it was inhibited in glioma-infiltrating microglia. In this study, the phagocytotic activity of microglia was facilitated by IL-12, which raises the possibility of success in cancer therapy via IL-12-modulated microglia.

In conclusion, AAV2 is an effective vehicle to transfer therapeutic genes in anti-GBM therapy. Among the defensive bioactivities of microglia, phagocytosis and the TRAIL death pathway were documented to be induced by AAV2mediated IL-12. The cytotoxicity of microglia on GBM cells was confirmed to be significantly increased via the stimulation of AAV2-mediated IL-12 through the TRAIL death pathway and phagocytosis. In summary, microglial cells can be modulated by AAV2-mediated IL-12 to increase anticancer activity and play an important role in the therapy of malignant brain tumors.

\section{References}

1. Portielje J, Gratama J, van Ojik H, Stoter G and Kruit W: IL-12: a promising adjuvant for cancer vaccination. Cancer Immunol Immunother 52: 133-144, 2003.

2. Fujiwara $\mathrm{H}$ and Hamaoka T: Antitumor and antimetastatic effects of interleukin 12. Cancer Chemoth Pharmacol 38: S22-S26, 1996.

3. Fehniger T, Cooper M and Caligiuri M: Interleukin-2 and interleukin-15: immunotherapy for cancer. Cytokine Growth Factor Rev 13: 169-183, 2002.

4. Dredge K, Marriott J, Todryk S and Dalgleish A: Adjuvants and the promotion of Th1-type cytokines in tumour immunotherapy. Cancer Immunol Immunother 51: 521-531, 2002.

5. Trinchieri G: Interleukin-12 and the regulation of innate resistance and adaptive immunity. Nat Rev Immunol 3: 133-146, 2003.

6. Colombo $\mathrm{M}$ and Trinchieri G: Interleukin-12 in anti-tumor immunity and immunotherapy. Cytokine Growth Factor Rev 13: 155-168, 2002.

7. Leonard JP, Sherman ML, Fisher GL, et al: Effects of singledose interleukin-12 exposure on interleukin-12-associated toxicity and interferon-gamma production. Blood 90: 2541-2548, 1997.

8. Kowalczuk A, Macdonald R, Amidei C, et al: Quantitative imaging study of extent of surgical resection and prognosis of malignant astrocytomas. Neurosurgery 41: 1028-1038, 1997.

9. Daneyemez M, Gezen F, Canakci Z and Kahraman S: Radical surgery and reoperation in supratentorial malignant glial tumors. Minim Invasive Neurosurg 41: 209-213, 1998.

10. Kayama T, Kumabe T, Tominaga T and Yoshimoto T: Prognostic value of complete response after the initial treatment for malignant astrocytoma. Neurol Res 18: 321-324, 1996.

11. Warrington $\mathrm{K}$ and Herzog R: Treatment of human disease by adeno-associated viral gene transfer. Hum Genet 119: 571-603, 2006.

12. Foti S, Haberman R, Samulski R and McCown T: Adenoassociated virus-mediated expression and constitutive secretion of NPY or NPY13-36 suppresses seizure activity in vivo. Gene Ther 14: 1534-1536, 2007.

13. Lo W, Qu G, Sferra T, Clark R, Chen R and Johnson P: Adenoassociated virus-mediated gene transfer to the brain: duration and modulation of expression. Hum Gene Ther 10: 201-213, 1999.

14. Flotte T: Gene therapy progress and prospects: recombinant adeno-associated virus (rAAV) vectors. Gene Ther 11: 805-810, 2004.

15. Aitken M, Moss R, Waltz D, et al: A phase I study of aerosolized administration of $\operatorname{tg}$ AAVCF to cystic fibrosis subjects with mild lung disease. Hum Gene Ther 12: 1907-1916, 2001.

16. Kaplitt M, Feigin A, Tang C, et al: Safety and tolerability of gene therapy with an adeno-associated virus (AAV) borne GAD gene for Parkinson's disease: an open label, phase I trial. Lancet 369: 2097-2105, 2007. 
17. Mittelbronn M, Dietz K, Schluesener H and Meyermann R: Local distribution of microglia in the normal adult human central nervous system differs by up to one order of magnitude. Acta Neuropathol 101: 249-255, 2001.

18. Hanisch U and Kettenmann H: Microglia: active sensor and versatile effector cells in the normal and pathologic brain. Nat Neurosci 10: 1387-1394, 2007.

19. Badie B and Schartner J: Flow cytometric characterization of tumor-associated macrophages in experimental gliomas. Neurosurgery 46: 957-962, 2000.

20. Kim S and de Vellis J: Microglia in health and disease. J Neurosci Res 81: 302-313, 2005.

21. Rock RB, Gekker G, Hu S, et al: Role of microglia in central nervous system infections. Clin Microbiol Rev 17: 942-964, 2004.

22. Hussain SF, Yang D, Suki D, Aldape K, Grimm E and Heimberger AB: The role of human glioma-infiltrating microglia/ macrophages in mediating antitumor immune responses. Neurooncology 8: 261-279, 2006.

23. Sliwa M, Markovic D, Gabrusiewicz K, et al: The invasion promoting effect of microglia on glioblastoma cells is inhibited by cyclosporin A. Brain 130: 476-489, 2007.

24. Watters J, Schartner J and Badie B: Microglia function in brain tumors. J Neurosci Res 81: 447-455, 2005.

25. Chiu T, Lin S, Hsieh W and Peng C: AAV2-mediated interleukin-12 in the treatment of malignant brain tumors through activation of NK cells. Int J Oncol 35: 1361-1367, 2009.

26. Wang M, Huang $H$, Hsieh S, Jeng $K$ and Kuo J: Resveratrol inhibits interleukin- 6 production in cortical mixed glial cells under hypoxia/hypoglycemia followed by reoxygenation. J Neuroimmunol 112: 28-34, 2001.

27. Feigin A, Kaplitt MG, Tang C, et al: Modulation of metabolic brain networks after subthalamic gene therapy for Parkinson's disease. Proc Natl Acad Sci USA 104: 19559-19564, 2007.
28. Cucchiarini M, Ren X, Perides G and Terwilliger E: Selective gene expression in brain microglia mediated via adeno-associated virus type 2 and type 5 vectors. Gene Ther 10: 657-667, 2003.

29. Shevtsova Z, Malik JMI, Michel U, Bähr M and Kügler S: Promoters and serotypes: targeting of adeno-associated virus vectors for gene transfer in the rat central nervous system in vitro and in vivo. Exp Physiol 90: 53-59, 2005

30. Wesolowska A, Kwiatkowska A, Slomnicki L, et al: Microgliaderived TGF-beta as an important regulator of glioblastoma invasion-an inhibition of TGF-beta-dependent effects by shRNA against human TGF-beta type II receptor. Oncogene 27: 918-930, 2008 .

31. Hussain S, Yang D, Suki D, Grimm E and Heimberger A: Innate immune functions of microglia isolated from human glioma patients. J Transl Med 4: 15, 2006.

32. Huang Y, Erdmann N, Peng H, Zhao Y and Zheng J: The role of TNF related apoptosis-inducing ligand in neurodegenerative diseases. Cell Mol Immunol 2: 113-122, 2005.

33. Aktas O, Schulze-Topphoff U and Zipp F: The role of TRAIL/ TRAIL receptors in central nervous system pathology. Front Biosci 12: 2912-2921, 2007.

34. Genc S, Kizildag S, Genc K, Ates H, Atabey N and Kizyldag S: Interferon gamma and lipopolysaccharide upregulate TNF-related apoptosis-inducing ligand expression in murine microglia. Immunol Lett 85: 271-274, 2003.

35. Johnstone R, Frew A and Smyth M: The TRAIL apoptotic pathway in cancer onset, progression and therapy. Nat Rev Cancer 8: 782-798, 2008 